\title{
DIASTOLIC DYSFUNCTION AND DIASTOLIC HEART FAILURE
}

Adelino F Leite-Moreira

Heart 2006;92:712-718. doi: 10.1136/hrt.2005.062950

Take the online multiple choice questions associated with this article (see page 711)
Correspondence to: Professor Adelino F LeiteMoreira, Department of Physiology, Faculty of Medicine, University of Porto, Alameda Professor Hernâni Monteiro, 4200-319 Porto, Portugal; amoreira@ med.up.pt $\int$ iastolic heart failure (HF) has emerged over the last two decades as a separate clinical entity. Approximately half of the patients presenting with symptoms of congestive HF exhibit a near normal left ventricular (LV) systolic function at rest, which is thought to be caused by a predominant abnormality in diastolic function. It is generally considered to have a somewhat better prognosis than systolic HF, but frequency of hospitalisations is comparable in systolic and diastolic HF. ${ }^{1}$ Prevalence of diastolic HF increases with age and is higher in women. It is associated with hypertension, hypertrophy, diabetes, ageing and ischaemia. ${ }^{2}$ Despite the recognition of its importance, definition and diagnostic criteria of diastolic dysfunction and diastolic HF remain controversial.

\section{DEFINITIONS}

The cardiac cycle encompasses systole and diastole. Regardless of the exact time limits of these periods, it is generally accepted that systolic function relates with the ability of the ventricle to contract and eject, while diastolic function reflects its ability to relax and fill. Diastolic dysfunction therefore refers to a disturbance in ventricular relaxation, distensibility or filling-regardless of whether the ejection fraction (EF) is normal or depressed and whether the patient is asymptomatic or symptomatic. ${ }^{3}$ If a patient, with preserved EF and diastolic dysfunction, exhibits symptoms of effort intolerance and dyspnoea, especially if there were evidence of venous congestion and oedema, the term diastolic HF is used. ${ }^{4}$

\section{PHYSIOLOGY OF DIASTOLIC FUNCTION}

Determinants of diastolic function (table 1) include myocardial relaxation and passive properties of the ventricular wall, such as myocardial stiffness, wall thickness and chamber geometry (size or volume). Other determinants include the structures surrounding the ventricle, the left atrium, pulmonary veins and mitral valve, and heart rate. Except for heart rate these other determinants are extrinsic to the ventricle and therefore normally not considered as true causes of ventricular diastolic dysfunction or failure. Moreover, diagnosis of diastolic HF implies exclusion of these determinants as the cause of ventricular filling disturbances.

\section{Relaxation}

Relaxation is the process whereby the myocardium returns to an unstressed length and force. In the normal heart it comprises the major part of ventricular ejection, pressure fall and the initial part of rapid filling. LV pressure fall is therefore the haemodynamic manifestation of myocardial relaxation and its analysis allows adequate description of the course of myocardial relaxation (see later).

Myocardial relaxation is modulated by load, inactivation and non-uniformity. ${ }^{5}$ Effects of load on relaxation depend on its type (preload versus afterload), magnitude, duration and timing in the cardiac cycle at which it occurs. ${ }^{6}$ When imposed early in the cardiac cycle a mild to moderate afterload elevation will, in the normal heart, delay the onset and accelerate the rate of pressure fall (compensatory response). On the contrary, a severe afterload elevation or an afterload elevation that occurs later in ejection will induce a premature onset and a pronounced slowing of pressure fall, even in a healthy heart (decompensatory response). Such slowing might lead to incomplete relaxation and therefore to elevation of filling pressures, a phenomenon that is exacerbated when preload is elevated. ${ }^{7}$ As pronounced hypertension represents a heavy afterload to the LV, this mechanism might contribute to exacerbation of diastolic dysfunction and congestion in acute hypertensive crisis. ${ }^{8}$

Myocardial inactivation relates to the processes underlying calcium extrusion from the cytosol and cross-bridge detachment. Determinants of myocardial inactivation, listed in table 2, therefore include mechanisms related to calcium homeostasis and myofilament regulators of cross-bridge cycling. Decreased concentrations or activity of the sarcoplasmic reticulum calcium ATPase pump (SERCA) can slow the removal of calcium from the cytosol. Increased levels or activity of 
Table 1 Determinants of diastolic function

Myocardial relaxation
- load
- inactivation (calcium homeostasis, myofilaments, energetics)
- non-uniformity
Passive properties of ventricular wall
- myocardial stiffness (cytoskeleton, extracellular matrix)
- wall thickness
- chamber geometry
Other determinants
- structures surrounding the ventricle (pericardium, lungs, remaining
- lefrd atrium, pulmonary veins and mitral valve
- heart rate

phospholamban, a SERCA-inhibitory protein, can also impair relaxation. Increased CAMP, resulting from $\beta$ adrenergic stimulation or inhibition of cardiac phosphodiesterase, phosphorylates phospholamban to remove its inhibitory effect on SERCA. The net effect is an improvement in diastolic relaxation. Pathological LV hypertrophy secondary to hypertension or aortic stenosis results in decreased SERCA and increased phospholamban, again leading to impaired relaxation. Similar changes are seen in the myocardium of patients with hypertrophic or dilated cardiomyopathy. Interestingly, concentrations of SERCA decrease with age, coincident with impaired diastolic function. ${ }^{1}$ As ATP hydrolysis is required for myosin detachment from actin, calcium dissociation from $\mathrm{Tn}-\mathrm{C}$, and active sequestration of calcium by the SR, energetic factors must also be taken in consideration. Modification of any of these steps, the myofilament proteins involved in these steps, or the ATPase that catalyses them can alter diastolic function. ${ }^{9}$ It is therefore not surprising that ischaemia leads to impaired relaxation.

During isovolumetric relaxation, re-extension of one ventricular segment is accompanied by post-systolic shortening of another segment. The ventricle remains isovolumic but changes its shape and produces intraventricular volume displacement. Asynchronous early segment re-extension and regional non-uniformity induce early onset and slower rate of ventricular pressure fall $^{56}$ and might contribute to the diastolic disturbances observed in coronary heart disease and with intraventricular conduction disturbances.

\section{Passive properties}

Passive properties of the ventricular wall are influenced by myocardial stiffness, wall thickness and chamber geometry. Determinants of myocardial stiffness include factors intrinsic to the cardiomyocytes themselves (cytoskeleton) and the extracellular matrix (ECM). The cardiomyocyte cytoskeleton is composed of microtubules, intermediate filaments (desmin), microfilaments (actin), and endosarcomeric proteins (titin, $\alpha$-actinin, myomesin, and M-protein). Changes in some of these cytoskeletal proteins have been shown to alter diastolic function. ${ }^{9}$

Most of the elastic force of the cardiomyocytes is now thought to reside in the macromolecule titin, whereas contributions of microtubules (tubulin) and intermediate filaments (desmin) appear $<10 \%$ at operating sarcomere lengths. ${ }^{10}$ Titin is expressed as varying isoforms that impart different mechanical properties, and this likely plays a role in altering passive stiffness in failing hearts. Titin can also be
Table 2 Determinants of myocardial inactivation

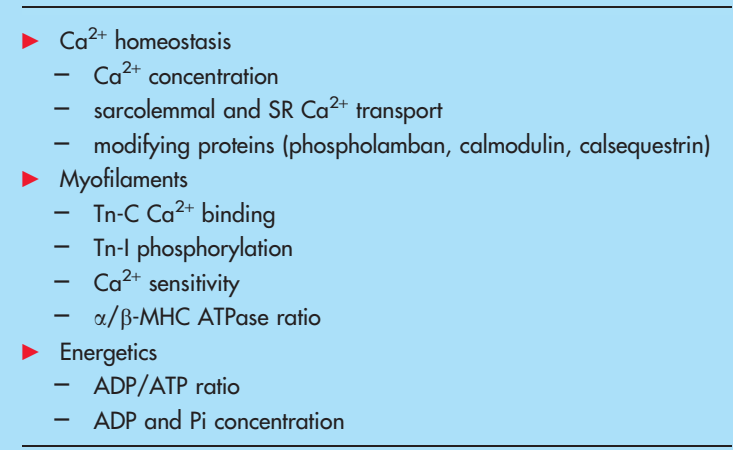

ADP, adenosine diphosphate; ATP, adenosine triphosphate; $M H C$ myosin heavy chain; SR, sarcoplasmic reticulum; Tn, troponin.

post-translationally modified by $\mathrm{Ca}^{2+}$ (even in the diastolic range) and by phosphorylation, blurring notions of passive versus active tone. ${ }^{10}$ Phosphorylation of sarcomeric proteins by pKA was recently shown to normalise increased stiffness of cardiomyocytes from patients with diastolic HF. ${ }^{11}$

Changes in the structures within the ECM can also affect diastolic function. Myocardial ECM is composed of: (1) fibrillar protein (for example, collagen types I and III, and elastin); (2) proteoglycans; and (3) basement membrane proteins (for example, collagen type IV, laminin, and fibronectin). Fibrillar collagen apparently is the most important component within the ECM contributing to the development of diastolic HF. ${ }^{9}$ The role played by other fibrillar proteins (basement membrane proteins and proteoglycans) remains largely unexplored.

ECM fibrillar collagen, particularly in terms of its amount, geometry, distribution, degree of cross-linking, and ratio of collagen type I/type III, is often altered in disease processes that alter diastolic function. The regulatory control of collagen biosynthesis and degradation includes: (1) transcriptional regulation by physical (for example, preload and afterload), neurohumoral (for example, renin-angiotensinaldosterone and sympathetic nervous systems), and growth factors; (2) post-translational regulation, including collagen cross-linking; and (3) enzymatic degradation. Collagen degradation is under the control of matrix metalloproteinases (MMPs). ${ }^{9}$ Changes in either synthesis or degradation and their regulatory processes have been shown to alter diastolic function and lead to the development of diastolic HF. In addition, it is now increasingly recognised that quality of collagen (specifically cross-linking and glycation) plays a key role in translating quantity into myocardial stiffness. ${ }^{10}$ Recent demonstration that 16 weeks of treatment with a glucose cross-link breaker decreased LV mass and improved diastolic filling and quality of life in patients with diastolic $\mathrm{HF}^{12}$ further reinforces this view.

In addition to post-translational modifications of titin, other evidence suggests that diastolic stiffness is actively modulated. Cross-bridge interaction occurs even at low diastolic calcium producing resting muscle tone. Modifications of myofilament calcium sensitivity by HF might also alter active tone. ${ }^{10}$ This includes changes associated with PKA (or PKG) phosphorylation of myosin light chain 2 and TnI. In this setting, nitric oxide (NO) and cyclic guanosine monophosphate (cGMP) increase resting diastolic cell length as a result of PKG-mediated phosphorylation of myofilaments, and in patients with dilated 
cardiomyopathy administration of intracoronary substance $\mathrm{P}$ (NO stimulator) decreases LV stiffness. ${ }^{7}$ Furthermore, myocardial stiffness is modulated by load and endothelin-1 (ET-1). Load elevations acutely increase LV stiffness, ${ }^{10}$ while ET- 1 acutely decrease myocardial stiffness. ${ }^{13}$

Heart rate

Heart rate (HR) influences myocardial oxygen demand and coronary perfusion time. Rapid HR increases the former and decreases the latter, so that ischaemic diastolic dysfunction might occur even in the absence of coronary disease, especially in hypertrophic hearts. Additionally, fast HR may shorten diastole to an extent that prevents relaxation from being complete between beats, resulting in increased filling pressures and therefore diastolic dysfunction. This may occur at lower HR in failing hearts, which contrary to normal hearts, may exhibit a flat or even negative relaxation velocityversus-HR relationship, so that as HR increases, relaxation rate does not increase or even decreases. ${ }^{9}$

\section{EVALUATION OF DIASTOLIC FUNCTION Relaxation}

As outlined above, ventricular pressure fall is the haemodynamic manifestation of myocardial relaxation. Its analysis includes measurement of rate and timing of its onset in the cardiac cycle (fig 1, left panel). Timing of LV pressure (LVP) fall can be estimated from the time interval between end diastole and $\mathrm{dP} / \mathrm{dt}_{\text {min }}$ or the time between aortic valve opening and closure (LVET). Indices of rate of LVP fall include $\mathrm{dP} / \mathrm{dt}_{\min }$, isovolumic relaxation time (IRT) and the time constant $\tau .^{6} \mathrm{DP} / \mathrm{dt}_{\min }$ is an instantaneous value that reflects maximal velocity of LVP fall. IRT is the time interval between aortic valve closure or $\mathrm{dP} / \mathrm{dt}_{\min }$ and mitral valve opening. It is influenced not only by relaxation rate but also by LVP at aortic valve closure and mitral valve opening. The time constant of isovolumic relaxation, tau $(\tau, \mathrm{ms})$, is the most widely used index to evaluate the rate of LVP fall. Tau is

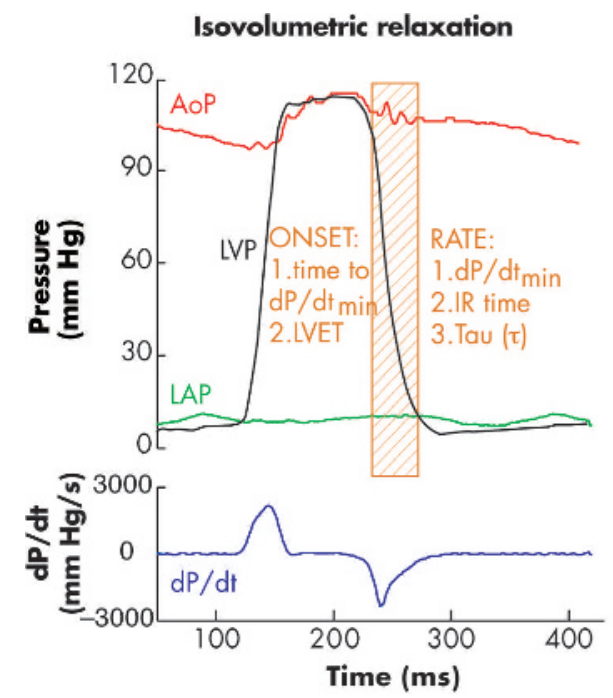

inversely related to the rate of LVP fall, becoming shorter when LVP fall accelerates and longer when LVP fall slows. Most methods assume that LVP fall follows a monoexponential course and can be described with the formula: $\mathrm{P}_{\mathrm{t}}=\left(\mathrm{P}_{0}-\mathrm{P}_{\infty}\right) \mathrm{e}^{-\mathrm{t} / \tau}+\mathrm{P}_{\infty}$ where $\mathrm{P}_{\mathrm{t}}$ is LVP at time $\mathrm{t} ; \mathrm{P}_{0}$ is LVP at $\mathrm{dP} /$ $\mathrm{dt}_{\text {min }}$ (time 0 ). $\mathrm{P}_{\infty}$ is the asymptotic pressure, to which relaxation would lead if completed without LV filling. $\mathrm{P}_{\infty}$ is negative in normal ventricles, which means that the nonfilling ventricle develops diastolic suction. According to this formula, $\tau$ corresponds to the time it takes for LVP to fall to 1/e $(\sim 36 \%)$ of its initial value. The formula also indicates that LVP fall, and therefore relaxation, will be $97 \%$ complete $3.5^{*} \tau$ after $\mathrm{dP} / \mathrm{dt}_{\min } \cdot{ }^{6}$

\section{Passive properties}

Chamber stiffness can be quantified by examination of the end diastolic pressure-volume relationship (EDPVR). This relationship is drawn by fitting the lower right corner of multiple pressure-volume (PV) loops obtained at various preloads to an exponential curve (fig 1, right panel). The operating stiffness at any point along a given EDPVR is equal to the slope of a tangent drawn to the curve at that point $(\Delta \mathrm{P} /$ $\Delta \mathrm{V})$. Operating stiffness changes with filling; stiffness is lower at smaller volumes and higher at larger volumes (volume dependent change in diastolic pressure and stiffness). As EDPVR is exponential, the relationship between $\Delta \mathrm{P} /$ $\Delta \mathrm{V}$ and pressure is linear. The slope $(\mathrm{Kc})$ of this line is the chamber stiffness constant and can be used to quantify chamber stiffness. ${ }^{4}$ When overall chamber stiffness is increased, the pressure-volume curve is shifted upwards and to the left, the slope of the $\Delta \mathrm{P} / \Delta \mathrm{V}$-versus-pressure relationship becomes steeper, and Kc is increased (volume independent change in diastolic pressure and stiffness). Thus, diastolic pressure can be changed either by a volume dependent change in operating stiffness or by a volume independent change in chamber stiffness.

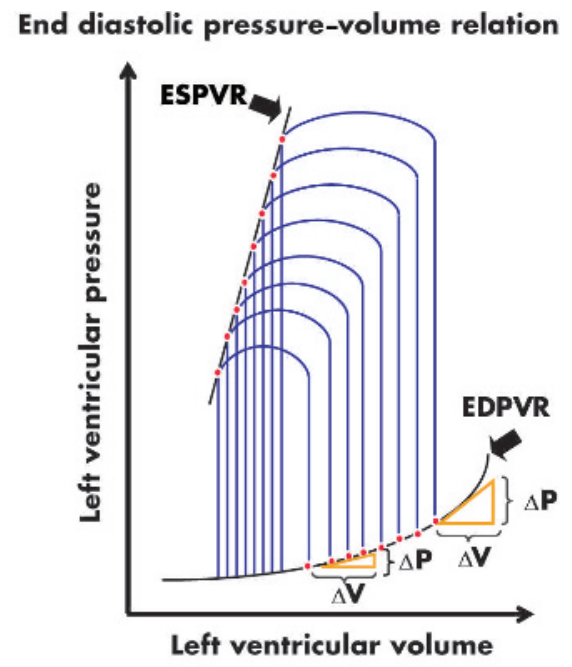

Figure 1 Haemodynamic evaluation of diastolic function. Left panel: Analysis includes rate and timing of onset of left ventricular (LV) pressure fall in the cardiac cycle. AoP, LVP, LAP, aortic, left ventricular, and left atrial pressures, respectively; $\mathrm{dP} / \mathrm{dt}$, first derivative of $\mathrm{LVP} ; \mathrm{dP} / \mathrm{dt}_{\mathrm{min}}$, peak rate of $\mathrm{LVP}$ fall; IR, isovolumetric relaxation. Right panel: LV pressure-volume loops at various preload and afterload levels. The end systolic pressure-volume relation (ESPVR) is used to evaluate LV contractility, while the end diastolic pressure-volume relation (EDPVR) allows quantification of chamber stiffness. The operating stiffness at any point along a given EDPVR is equal to the slope of a tangent drawn to the curve at that point $(\Delta P / \Delta V)$. Operating stiffness changes with filling; stiffness is lower at smaller volumes and higher at larger volumes (volume dependent change in diastolic pressure and stiffness). 

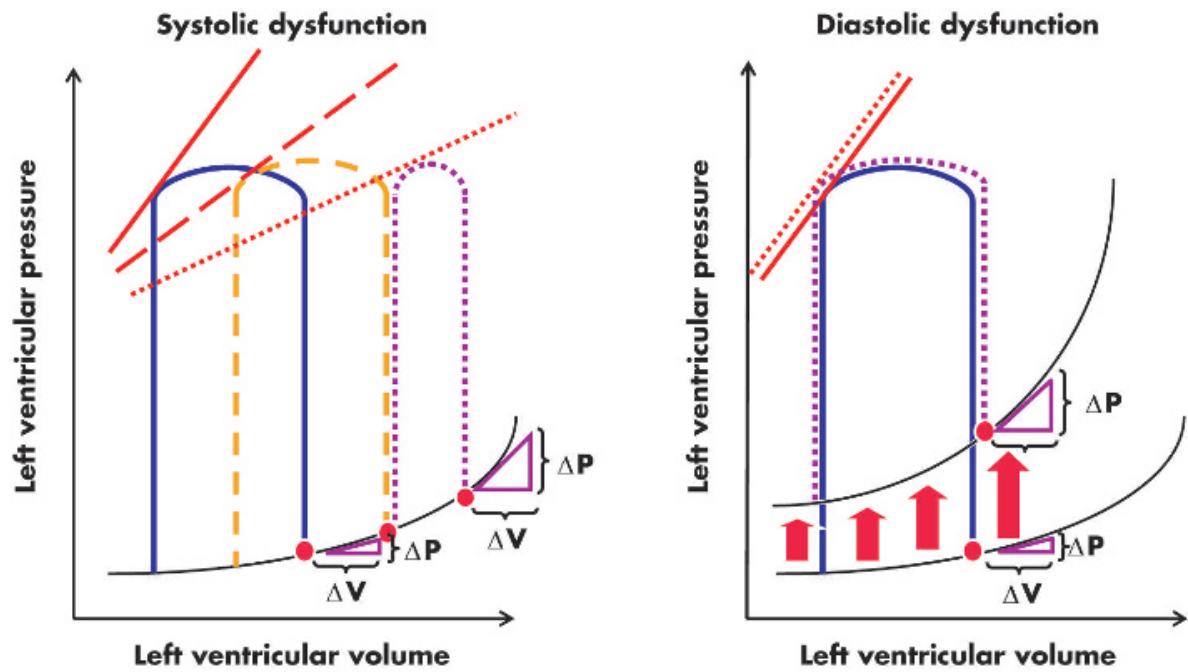

Figure 2 Effects of systolic dysfunction (left panel) and diastolic dysfunction (right panel) on pressure-volume (PV) loops, and end systolic (ESPVR) and end diastolic PV relations (EDPVR). In both panels the blue PV loops represent cardiac cycles from a normal heart. When systolic dysfunction develops the ventricle uses preload reserve in an attempt to preserve ejection fraction (dashed orange line). When systolic dysfunction progresses, ejection fraction (PV loop width) cannot be maintained even if end diastolic pressures are further increased (dotted purple line, left panel). Therefore, in systolic dysfunction increased filling pressures reflect a rightward shift along the same EDPVR and a volume dependent increase in operating stiffness. On the contrary, when diastolic dysfunction occurs, ejection fraction is preserved and end diastolic pressures are raised due to a left and upward shift of the entire PV relation, reflecting a volume independent increase in chamber stiffness. Note that the slope of the ESPVR (red lines) progressively decreases with systolic dysfunction, but is preserved in diastolic dysfunction.

Figure 2 shows that while in systolic dysfunction (left panel) end diastolic pressures are usually elevated by a volume dependent increase in operating stiffness, in diastolic dysfunction (right panel) end diastolic pressures are elevated by a volume independent increase in chamber stiffness. In both panels the blue PV loops represent a cardiac cycle from a normal heart. When systolic dysfunction develops the ventricle uses preload reserve to preserve EF (dashed orange line). When systolic dysfunction progresses, EF (PV loop width) cannot be maintained anymore, even if end diastolic pressures are further increased (dotted purple line). Therefore, in systolic dysfunction increased filling pressures reflect a rightward shift along the same EDPVR and a volume dependent increase in operating stiffness. It should be noted that when ventricular dilatation occurs, the entire EDPVR is right and downward shifted and therefore a volume dependent increase in operating stiffness might occur even if volume independent chamber stiffness is decreased. On the contrary, when diastolic dysfunction occurs EF is preserved and end diastolic pressures are raised due to a left and upward shift of the entire PV relation, reflecting a volume independent increase in chamber stiffness. Note that the slope of the end systolic PV relation (red lines) progressively decreases with systolic dysfunction, but is preserved in diastolic dysfunction.

\section{Echocardiographic indices}

In addition to providing information about LV dimensions and EF, both important for the diagnosis of diastolic HF, echocardiography provides indices of diastolic function. ${ }^{1}$ The most familiar of these are the mitral inflow velocities; the $\mathrm{E}$ and A waves that correspond, respectively, to early flow during LV relaxation and subsequent contribution of atrial contraction. When diastolic function is normal $\mathrm{E}$ wave exceeds A wave velocity. Pulmonary vein flow is also measured in two phases, systolic and diastolic, analogous to the $\mathrm{x}$ and $\mathrm{y}$ descents of the jugular veins.

With impaired relaxation, atrial contraction contributes relatively more to ventricular filling $(\mathrm{A}>\mathrm{E}$, with prolonged deceleration of the $\mathrm{E}$ wave). This state is common with increasing age and may identify patients at risk for diastolic HF. When LV diastolic pressure increases to the point that atrial contraction contributes little to filling, the E wave again becomes predominant but with rapid deceleration, first in a pseudonormal pattern and ultimately in a restrictive pattern (high E wave velocity, usually more than twice the A wave velocity) (fig 3). Both filling patterns are reliably associated with diastolic dominant pulmonary vein flow in patients over 50 years of age. ${ }^{1}$

New indices derived from transmitral flow propagation (Vp) and from tissue Doppler velocity (Em) are less sensitive to load and provide new tools to detect early abnormalities or to study non-invasively the effects of new therapies on diastolic function. For a recent, extensive review on noninvasive assessment of diastolic function see Quinones. ${ }^{14}$

\section{DIAGNOSIS}

The European Society of Cardiology study group on diastolic HF proposed three obligatory criteria that should be met simultaneously for the diagnosis ${ }^{15}$ : (1) presence of signs or symptoms of congestive HF; (2) normal or only mildly abnormal LV systolic function; and (3) evidence of abnormal LV relaxation, filling, diastolic distensibility or diastolic stiffness. Several criticisms were made to these criteria. First, the requirement of signs or symptoms, instead of signs and symptoms, for the clinical diagnosis of CHF (fulfilment of Framingham criteria had been suggested). Second, the cut off value of $45 \%$ for LVEF. Although values in the $40-50 \%$ range were used in several studies, Vasan and Levy ${ }^{16}$ proposed an LVEF $>50 \%$. These authors developed criteria 


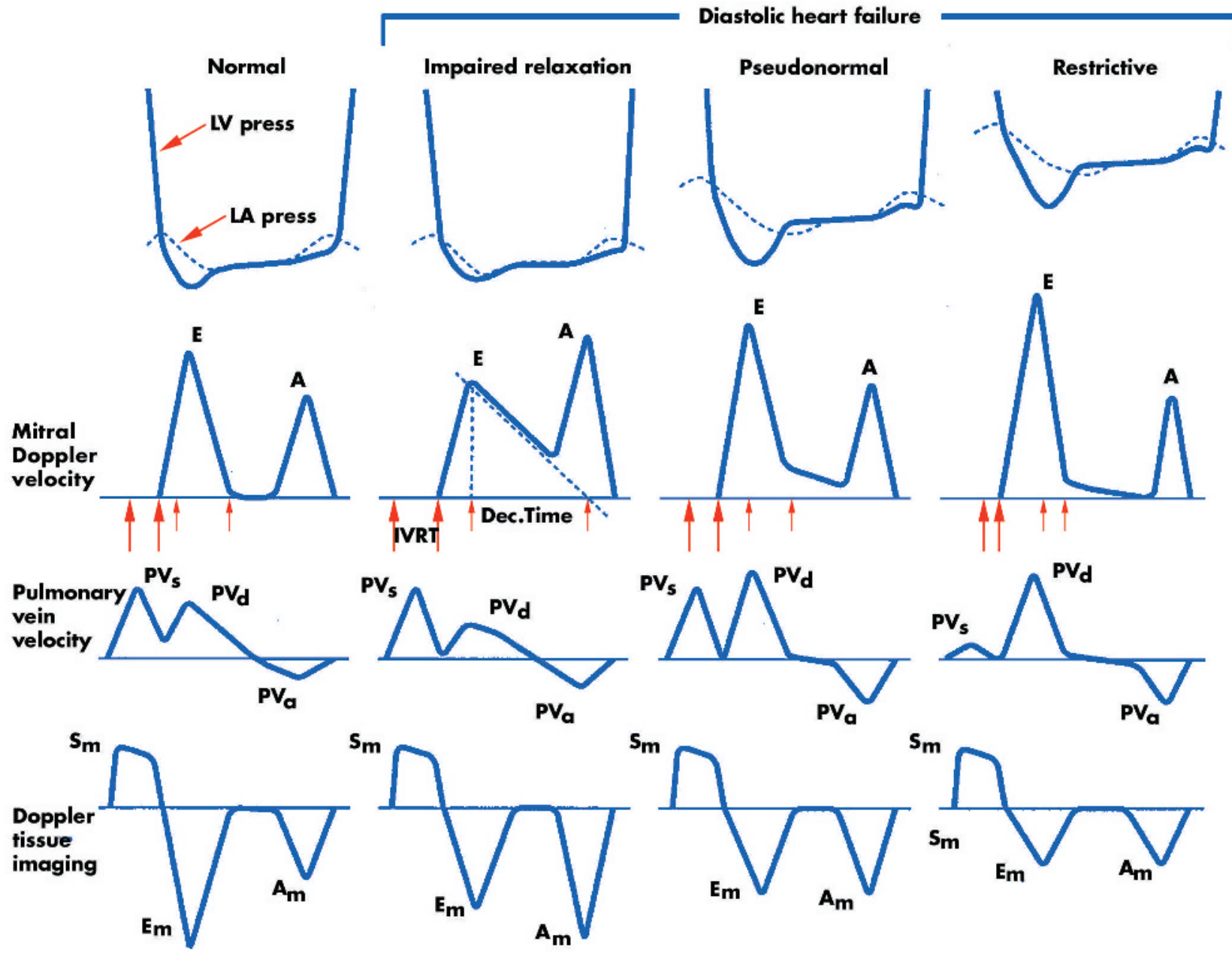

Figure 3 Left ventricular (LV) and left atrial (LA) pressures during diastole, transmitral Doppler LV inflow velocity, pulmonary vein Doppler velocity, and Doppler tissue velocity. A, velocity of LV filling contributed by atrial contraction; Am, myocardial velocity during filling produced by atrial contraction; Dec. Time, e-wave deceleration time; E, early LV filling velocity; Em, myocardial velocity during early filling; IVRT, isovolumic relaxation time; PVa, pulmonary vein velocity resulting from atrial contraction; PVd, diastolic pulmonary vein velocity; PVs, systolic pulmonary vein velocity; Sm, myocardial velocity during systole. Reproduced from Zile and Brutsaert, ${ }^{9}$ with permission.

for definite, probable, and possible diastolic HF. Definite diastolic HF requires definitive evidence of HF, LVEF > 50\% (evaluated less than 72 hours after the HF event), and evidence by cardiac catheterisation (the European study group also accepts echocardiography) of abnormal LV relaxation, filling, diastolic distensibility, or diastolic stiffness. If catheterisation evidence of diastolic dysfunction is not available they propose a diagnosis of probable diastolic $H F$, or of possible diastolic HF if LVEF is measured more than 72 hours after the HF event. In fact, LVEF can vary according to when it is determined. For example, in HF secondary to acute transitory myocardial ischaemia or hypertensive crisis, LVEF determined during the first hours can be reduced but at 24 hours it is normal. However, Gandhi and colleagues ${ }^{8}$ showed that in patients with HF and uncontrolled hypertension differences between LVEF determined in the emergency department and at 72 hours were not significant in those patients who were already clinically stable. Thus, it is not usually essential to determine LVEF during initial decompensation as values obtained in the following days are reliable; the only exception to this rule may be in patients with acute ischaemia.
The clinical application of these criteria is limited because of their complexity and the fact that both demand demonstrable abnormalities in diastolic function. Recognising the difficulties inherent in the assessment of the diastolic properties of the heart, Zile and colleagues ${ }^{17}$ tested the hypothesis that measurements of the LV relaxation and passive stiffness were not necessary to make the diagnosis of diastolic HF. They showed that among patients with CHF diagnosed according to Framingham criteria and LVEF $>50 \%$ who undergo a haemodynamic study and Doppler echocardiogram, $100 \%$ present at least one diastolic abnormality identified by one or other of these methods. Consequently, the study of diastolic function serves to confirm the diagnosis of diastolic congestive HF rather than establish it, although a better characterisation of the underlying mechanism might help to guide treatment. Furthermore, it was recently shown that diastolic dysfunction (abnormal relaxation and passive stiffness) is the predominant pathophysiological cause of HF in patients who met the diagnostic criteria of definite diastolic HF. ${ }^{18}$

Increasing evidence suggest that B-type natriuretic peptide (BNP) and N-terminal pro-BNP (NT-proBNP) might 
facilitate the differential diagnosis of HF and help to distinguish patients with systolic from those with diastolic $\mathrm{HF}^{3}$

\section{PROGNOSIS}

Prognosis of diastolic HF is slightly less ominous than that of systolic HF, with an annual mortality of $5-8 \%$ in those individuals with the former and $10-15 \%$ in those with the latter. ${ }^{4}$ Mortality in the general population without HF and of a similar age is $1 \%$ per year. Presence of coronary disease, age and the LVEF cut-off value are important factors in the prognosis. When patients with ischaemic heart disease are excluded, annual mortality for diastolic congestive HF falls to $2-3 \%$. In patients $>70$ years with congestive HF, mortality is similar in systolic and diastolic HF. ${ }^{2}$

\section{TREATMENT}

To date, only one large scale monitored randomised clinical trial was undertaken to compare drug versus placebo administration in patients with $\mathrm{HF}$ and preserved systolic function (CHARM-preserved). This trial compared the efficacy of a daily $32 \mathrm{mg}$ dose of candesartan versus a placebo in 3023 patients with chronic HF and LVEF > 40\%. After a 36.6 month mean follow up, primary combined outcome incidence (death by cardiovascular cause or admission for congestive HF) was similar in both groups. Data for cardiovascular mortality did not differ, but a moderate impact of candesartan in preventing admissions for congestive HF among patients who have HF and LVEF > 40\% was observed. ${ }^{19}$

Although the moderate benefit of candesartan should be taken into consideration, until data from randomised clinical trials provide new evidence, Zile and Brutsaert ${ }^{9}$ propose that treatment of diastolic HF must be directed toward symptoms, aetiology and, in the future, underlying mechanisms, as

Table 3 Diastolic heart failure: treatment ${ }^{9}$

\begin{tabular}{|c|c|}
\hline \\
\hline \multicolumn{2}{|c|}{$\begin{array}{l}\text { Symptom targeted treatments } \\
\text { Decrease pulmonary venous pressure }\end{array}$} \\
\hline & reduce left ventricular volume \\
\hline & maintain atrial contraction \\
\hline 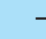 & prevent tachycardia \\
\hline$>1$ & nprove exercise tolerance \\
\hline$>1$ & se positive inotropic agents with caution \\
\hline$>1$ & lon-pharmacological treatment \\
\hline & restrict sodium to prevent volume overload \\
\hline & restrict fluid to prevent volume overload \\
\hline & $\begin{array}{l}\text { perform moderate aerobic exercise to improve cardiovascular } \\
\text { conditioning, decrease heart rate, and maintain skeletal muscle } \\
\text { function }\end{array}$ \\
\hline$>\mathrm{F}$ & harmacological treatment \\
\hline & diuretics, including loop diuretics, thiazides, spironolactone \\
\hline & long acting nitrates \\
\hline & $\beta$ adrenergic blockers \\
\hline & calcium channel blockers \\
\hline & $\begin{array}{l}\text { renin-angiotensin-aldosterone antagonists, including angiotensin } \\
\text { converting enzyme (ACE) inhibitors, angiotensin II receptor } \\
\text { blockers, and aldosterone antagonists }\end{array}$ \\
\hline Dis & ase targeted treatments \\
\hline$>\mathrm{F}$ & revent/treat myocardial ischaemia \\
\hline $\mathrm{Me}$ & $\begin{array}{l}\text { revent/regress ventricular hypertrophy } \\
\text { hanism targeted treatments }\end{array}$ \\
\hline$>1$ & lodify myocardial and extramyocardial mechanisms \\
\hline$>1$ & lodify intracellular and extracellular mechanisms \\
\hline
\end{tabular}

Diastolic dysfunction and heart failure: key

points

- Approximately half of the patients presenting with symptoms of congestive heart failure exhibit a near normal left ventricular (LV) systolic function at rest, which is thought to be caused by a predominant abnormality in diastolic function

- Prevalence of diastolic heart failure increases with age and is higher in women. It is associated with hypertension, hypertrophy, diabetes, ageing and ischaemia

- Mortality is somewhat lower but morbidity is similar in diastolic versus systolic heart failure

- Determinants of diastolic function include myocardial relaxation and passive properties of the ventricular wall

- Myocardial relaxation is modulated by load, inactivation and non-uniformity

- Passive properties of the ventricular wall are influenced by myocardial stiffness (cytoskeleton and extracellular matrix), wall thickness and chamber geometry

- There is now evidence that diastolic stiffness is actively modulated by load and neurohumoral agents

- The time constant of isovolumic relaxation, tau $(\tau)$, is the most widely used index to evaluate the rate of left ventricular pressure fall, which is the haemodynamic manifestation of myocardial relaxation

- Chamber stiffness can be quantified by examination of the end diastolic pressure-volume relationship

- Echocardiographic indices derived from Doppler mitral inflow velocities are load dependent, but this limitation might be compensated by new indices derived from pulmonary venous flow, transmitral flow propagation and tissue Doppler velocity

- Virtually all patients with congestive heart failure and a normal ejection fraction exhibit evidence of diastolic dysfunction

- Until data from randomised clinical trials provide new evidence, treatment of diastolic heart failure must be directed toward symptoms, aetiology and, in the future, underlying mechanisms

- Statin treatment may be associated with lower mortality in patients with diastolic heart failure

outlined in table 3. Aetiologic factors include hypertension, diabetes, or ischaemia. Angiotensin receptor blockers (ARBs) have proven effective in causing regression of LV hypertrophy (LIFE) and may reduce morbidity, but not mortality (CHARM). Maintenance of sinus rhythm, heart rate control ( $\beta$ blockers, calcium channel blockers) and anti-ischaemic treatment may be indicated in view of pathophysiological aspects. Diuretics should be administered with caution in patients with symptoms of congestion; digitalis is not useful in the treatment of isolated diastolic HF. The results of ongoing trials (for example, I-Preserve) may offer new therapeutic options, and evidence based guidelines for the so far often unsatisfactory treatment of diastolic dysfunction/ HF are awaited.

Therefore, even if the rationale of their use differs, these principles suggest that drugs recommended for diastolic HF may be the ones recommended for systolic dysfunction. For example, $\beta$ blockers are now recommended for the treatment of both systolic and diastolic HF. In diastolic HF, however, $\beta$ blockers are used to decrease heart rate, increase the duration of diastole, and modify the haemodynamic response to exercise. In systolic HF, $\beta$ blockers are used chronically to increase inotropic state and modify LV remodelling. In 
systolic HF, $\beta$ blockers must be titrated slowly and carefully over an extended time period. This is generally not necessary in diastolic HF. Diuretics are used in the treatment of both systolic and diastolic HF. However, the doses of diuretics used to treat diastolic HF are generally smaller than the doses used in systolic HF. Some drugs are used only to treat either systolic or diastolic HF, but not both. For example, calcium channel blockers have no place in the treatment of systolic HF, but have been considered potentially useful in the treatment of diastolic HF.'

Conceptually, an ideal therapeutic agent should target the underlying mechanisms that cause diastolic HF. Therefore, a therapeutic agent might improve calcium homeostasis and energetics, blunt neurohumoral activation and decrease myocardial stiffness. Fortunately, some pharmaceutical agents that fit these design characteristics are already in existence, and many more are under development. Unfortunately, randomised, double blind, placebo controlled, multicentre trials that examine the efficacy of these agents used either singly or in combination have been slow to develop.

Two recent pilot studies opened new perspectives for diastolic HF treatment. In the first one, 16 weeks treatment with ALT-711, a glucose crosslink breaker, resulted in a decrease in LV mass and improvements in LV diastolic filling and quality of life in elderly patients with diastolic HF. ${ }^{12}$ This drug targets increased myocardial stiffness caused by collagen cross-linking. This pathophysiological mechanism of diastolic HF was not tested previously in HF. The other study gathered evidence that statin treatment may be associated with lower mortality in patients with diastolic $\mathrm{HF}^{20}{ }^{20}$ by mechanisms that still remain speculative. This was the first study showing a beneficial effect of a drug on mortality of diastolic HF patients.

In compliance with EBAC/EACCME guidelines, all authors participating in Education in Heart have disclosed potential conflicts of interest that might cause a bias in the article

\section{REFERENCES}

1 Angeja BG, Grossman W. Evaluation and management of diastolic heart failure. Circulation 2003; 107:659-63.

2 Owan TE, Redfield MM. Epidemiology of diastolic heart failure. Prog Cardiovasc Dis 2005;47:320-32.

- Excellent review with emphasis on the epidemiology of diastolic heart failure.

3 Gaasch WH, Zile MR. Left ventricular diastolic dysfunction and diastolic heart failure. Annu Rev Med 2004;55:373-94.
4 Zile MR, Brutsaert DL. New concepts in diastolic dysfunction and diastolic heart failure. Part I: diagnosis, prognosis, and measurements of diastolic function. Circulation 2002;105:1387-93.

- First part of an excellent and comprehensive review about the basic and clinical features of diastolic dysfunction and failure.

5 Brutsaert DL, Sys SU. Relaxation and diastole of the heart. Physiol Rev 1989;69:1228-315

6 Leite-Moreira AF, Gillebert TC. The physiology of left ventricular pressure fall. Rev Port Cardiol 2000; 19:1015-21.

7 Leite-Moreira AF, Correia-Pinto J. Load as an acute determinant of enddiastolic pressure-volume relation. Am J Physiol Heart Circ Physiol 2001;280:H51-9

8 Gandhi SK, Powers JC, Nomeir AM, et al. The pathogenesis of acute pulmonary edema associated with hypertension. N Engl J Med 2001;344:17-22.

9 Zile MR, Brutsaert DL. New concepts in diastolic dysfunction and diastolic heart failure. Part II: causal mechanisms and treatment. Circulation 2002; 105:1503-8

- Second part of an excellent and comprehensive review about the basic and clinical features of diastolic dysfunction and failure.

10 Kass DA, Bronzwaer JG, Paulus WJ. What mechanisms underlie diastolic dysfunction in heart failure? Circ Res 2004;94:1533-42.

- Excellent review with emphasis on the basic mechanisms underlying diastolic dysfunction and failure.

11 Borbely A, van der Velden J, Papp Z, et al. Cardiomyocyte stiffness in diastolic heart failure. Circulation 2005;111:774-81.

12 Little WC, Zile MR, Kitzman DW, et al. The effect of alagebrium chloride (ALT 711), a novel glucose cross-link breaker, in the treatment of elderly patients with diastolic heart failure. J Card Fail 2005;11:191-5.

- Pilot study showing the benefits, in patients with diastolic heart failure of a cross-link breaker which influences a mechanism outside the classic therapeutic targets for systolic heart failure.

13 Leite-Moreira AF, Bras-Silva C, Pedrosa CA, et al. ET-1 increases distensibility of acutely loaded myocardium: a novel ETA and $\mathrm{Na}+/ \mathrm{H}+$ exchangermediated effect. Am J Physiol Heart Circ Physiol 2003;284:H1332-9.

14 Quinones MA. Assessment of diastolic function. Prog Cardiovasc Dis 2005:47:340-55

- Excellent review with emphasis on the non-invasive assessment of diastolic function.

15 European Study Group on Diastolic Heart Failure. How to diagnose diastolic heart failure. Eur Heart J 1998;19:900-1003.

- Landmark consensus document on the diagnostic criteria of diastolic heart failure.

16 Vasan RS, Levy D. Defining diastolic heart failure: a call for standardized diagnostic criteria. Circulation 2000;101:2118-21.

17 Zile MR, Gaasch WH, Carroll JD et al. Heart failure with a normal ejection fraction: is measurement of diastolic function necessary to make the diagnosis of diastolic heart failure? Circulation 2001;104:779-82.

- Landmark study showing that most patients with heart failure and normal ejection fraction have diastolic dysfunction.

18 Zile MR, Baicu CF, Gaasch WH. Diastolic heart failure-abnormalities in active relaxation and passive stiffness of the left ventricle. N Engl J Med 2004;350:1953-9

19 Yusuf S, Pfeffer MA, Swedberg K, et al for the CHARM Investigators and Committees. Effects of candesartan in patients with chronic heart failure and preserved left-ventricular ejection fraction: the CHARM-Preserved trial. Lancet 2003;362:777-81.

- First clinical trial showing the benefits of a pharmacologic intervention in patients with diastolic heart failure. Candesartan had a moderate impact in preventing admissions for CHF in these patients.

20 Fukuta H, Sane DC, Brucks S, et al. Statin therapy may be associated with lower mortality in patients with diastolic heart failure. A preliminary report. Circulation 2005; 112:357-63.

- Pilot study showing for the first time that a pharmacologic intervention might decrease mortality of patients with diastolic heart failure. 